\title{
Structure of a group related to a Napoleon tessellation
}

\author{
Vlastimil Dlab \\ Vlastimil Dlab started his mathematical career 1956 in algebra at the Charles Univer- \\ sity in Prague. Then, he had positions at the University of Khartoum, at the Institute of \\ Advanced Studies in Canberra, Australia, and finally at Carleton University in Ottawa, \\ Canada. He visited mathematics departments all around the world and was elected Fel- \\ low of the Royal Society of Canada in 1977. Presently he is Professor Emeritus and \\ Distinguished Research Professor at Carleton University and Professor Hospitus at \\ Charles University.
}

The presentation of a two-dimensional plane using the repetition of a geometric shape with no overlaps and no gaps, the so-called tessellation, or tiling, appears as a natural structure in the real world and is displayed frequently in arts since ancient times. It is the Moorish architecture in Alhambra, and, in modern times, the work of M. C. Escher that made this concept a familiar notion.

Here, we are interested in "periodic" tessellations, the beginnings of whose studies can be traced to their classification in terms of seventeen different groups of isometries by Evgraf Stepanovich Fedorov in [3]. This classification, rediscovered later by a number of algebraists, crystallographers and geometers, provides a beautiful bond between group theory and geometry of the plane. On the one hand, theory of groups furnishes an abstract description of all possible plane patterns in terms of their symmetries and on the other hand, a particular tessellation can render a complete structure of a group given by generators and relations. The present note relates to the latter category, viz. it describes the structure of a group given by a very simple presentation exploring its identification with the symmetry

In den kunstvoll gestalteten Ornamenten der Alhambra findet man alle Parkettierungsarten, welche den 17 ebenen kristallographischen Gruppen entsprechen. Der Zusammenhang zwischen diesen endlichen Gruppen und den entsprechenden Parkettierungen der Ebene ist schon lange bakannt und reflektiert die Maxime, dass Gruppen Symmetrien von Objekten darstellen. Inspiriert vom Satz von Napoleon werden NapoleonParkettierungen erzeugt von einem beliebigen Dreieck und den über dessen Seiten errichteten gleichseitigen Dreiecken. Sie liefern eine anschauliche Beschreibung der Gruppe $G=\left\langle a, b \mid a^{3}=b^{3}=(a b)^{3}=1\right\rangle$ : Die Gruppe wird durch ein Bild ersetzt, wodurch technische Argumente visuell illustriert werden können. 
group of a particular tessellation. The group in question is denoted by $\mathbf{p 3}$ in HermannMauguin notation that has been adopted by the International Union of Crystallography and used by Coxeter-Moser in their monograph [2].

The symmetry group $\mathbf{p 3}$ relates to a tessellation generated by a general (scalene) triangle using the Napoleon theorem construction. In the final remark of this note, we briefly discuss the special cases of triangles with additional symmetries.

Thus, we wish to present a comprehensive description of the structure of the following (polyhedral) group

$$
G=\left\langle a, b \mid a^{3}=b^{3}=(a b)^{3}=1\right\rangle
$$

using a geometric representation, and thus demonstrating the maxim that groups are symmetries of objects. The paper translate the group into a picture that allows to replace technical arguments with visual demonstrations. First, let us formulate the main theorem.

Theorem 1. The group defined by (*) is isomorphic to the semidirect product $H \rtimes K$, where $H$ is the free abelian group of rank 2 and $K$ the cyclic group of order $3: H=$ $\left\langle a^{2} b\right\rangle \times\left\langle b a^{2}\right\rangle$ and $K=\langle a\rangle$. The semidirect product is provided by the isomorphism $\varphi: K \rightarrow \operatorname{Aut}(H)$ defined by $\varphi_{a}\left(a^{2} b\right)=a b a$ and $\varphi_{a}\left(b a^{2}\right)=a^{2} b$.

Note that $a b a \in H$ since $a b a=a(b a)^{-2}=a\left(a^{2} b^{2}\right)^{2}=\left(b^{2} a\right)\left(a b^{2}\right)=\left(a^{2} b\right)^{-1}\left(b a^{2}\right)^{-1}$. It is also easy to check that $H$ is in fact abelian and normal in $G$. Moreover, $\left(a^{2} b\right)^{n} a=$ $a\left(a^{2} b\right)^{-n}\left(b a^{2}\right)^{-n}$ and $\left(b a^{2}\right)^{n} a=a\left(a^{2} b\right)^{n}$. In view of these simple identities, one can define for every element $g=a^{e_{1}} b^{e_{2}} a^{e_{3}} b^{e_{4}} \ldots a^{e_{d-1}} b^{e_{d}} \in G$ the weight $w$ by $w(g)=$ $\sum_{k=1}^{d} e_{k}$. Every word $g$ with weight $w(g)$ divisible by three is in $H$ and the index of $H$ in $G$ is thus at most three.

The proof of Theorem 1 follows immediately from the representation of $G$ as a symmetry group of a general Napoleon tessellation. This tessellation is related to the following theorem, often called the Napoleon Theorem (see e.g. Coxeter [1] or Rigby [4]): Given an arbitrary triangle $\triangle=A B C$, erect on its sides (externally) the equilateral triangles $\triangle_{1}=B P C, \triangle_{2}=C Q A$ and $\triangle_{3}=A R B$. Denote by $U, V$ and $W$ the centroids of these triangles. Then the triangle $\triangle_{0}=U V W$ is equilateral and its centroid $G$ coincides with the centroid of the original triangle $\Delta$ (see Figure 1).

The Napoleon Theorem provides immediately a (Napoleon) tessellation (wallpaper) indicated in Figure 2.

In particular, the centroids of the equilateral triangles define a new tessellation by equilateral triangles, all congruent to the triangle $\Delta_{0}$. This tessellation is indicated in Figure 2 by dotted lines. Note that six of such triangles with a common vertex (that is the centroid of one of the equilateral triangles of the Napoleon tessellation) form a regular hexagon.

In order to describe the geometric representation of the group $G$ given by (*) as a symmetry group of this tessellation consider Figure 3, where the points $U, V$ and $W$ are described by the "coordinates" $(0,1),(-1,1)$ and $(0,0)$, respectively. Let us point out that we are using the centroids of the equilateral triangles of the Napoleon tessellation to define a coordinate system (indicated in Figure 3 by two bold dotted lines) that is not cartesian and has different scales in the two chosen directions. This system enables us to define translations of the plane that will represent some elements of our group. 


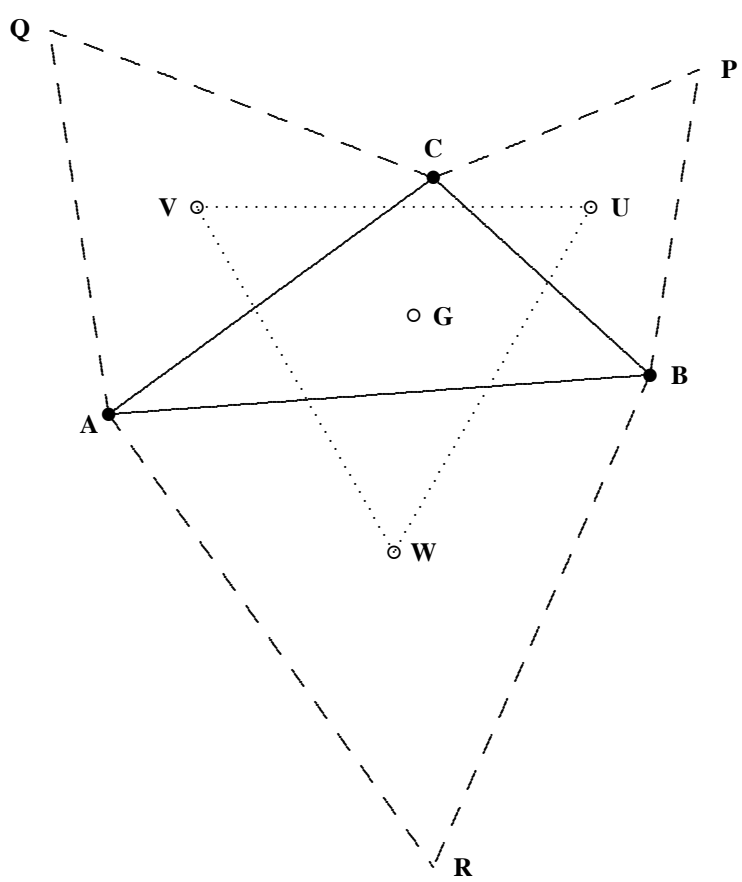

Figure 1

However, it will be rotations by $\frac{2 \pi}{3}$ that will play a fundamental role in the geometric representation of the group. Indeed, some elements of the group (and, the generators $a$ and $b$, in particular) will be represented by $\frac{2 \pi}{3}$ rotations along the dotted hexagons of the previous Figure 2. Here we deal with the original angles on the plane, rather than with some formal angles in the new coordinates. This fact is emphasized in Figure 3 by long dotted lines at angle $\frac{\pi}{3}$.

Thus, denote by $\mathbf{R}_{(r, s)}$ the (anticlockwise) rotations of the plane around ( $\left.r, s\right)$ by $\frac{2 \pi}{3}$. These are, for all $r, s \in \mathbf{Z}$, symmetries (of order 3 ) of the considered Napoleon tessellation. Moreover, denote by $\mathbf{T}_{(0,0) \rightarrow(r, r+3 t)}$ the translation of the plane mapping $(0,0)$ to $(r, r+3 t)$. Observe that for $r, t \in \mathbf{Z}$, these are all the translations of the plane that provide symmetries of the tessellation. Finally, denote by $\mathbf{S}_{N}$ the symmetry group of the given general Napoleon tessellation, i.e. the group generated by $\mathbf{R}_{(0,0)}, \mathbf{R}_{(0,1)}, \mathbf{R}_{(-1,0)}, \mathbf{T}_{(0,0) \rightarrow(1,-2)}, \mathbf{T}_{(0,0) \rightarrow(1,1)}$ and $\mathbf{T}_{(0,0) \rightarrow(2,-1)}$. Now, we can formulate the following theorem.

Theorem 2. The mapping $\psi: G \rightarrow \mathbf{S}_{N}$ given by

$$
\psi(a)=\mathbf{R}_{(0,0)} \text { and } \psi(b)=\mathbf{R}_{(0,1)}
$$

is an isomorphism of the groups $G$ and $\mathbf{S}_{N}$.

Proof. First, one can see easily that $\psi(a) \psi(b)=R_{(-1,1)}^{2}$ is of order three. Therefore, $\psi$ is a well-defined group epimorphism. The generators of $H$ are mapped to translations: 


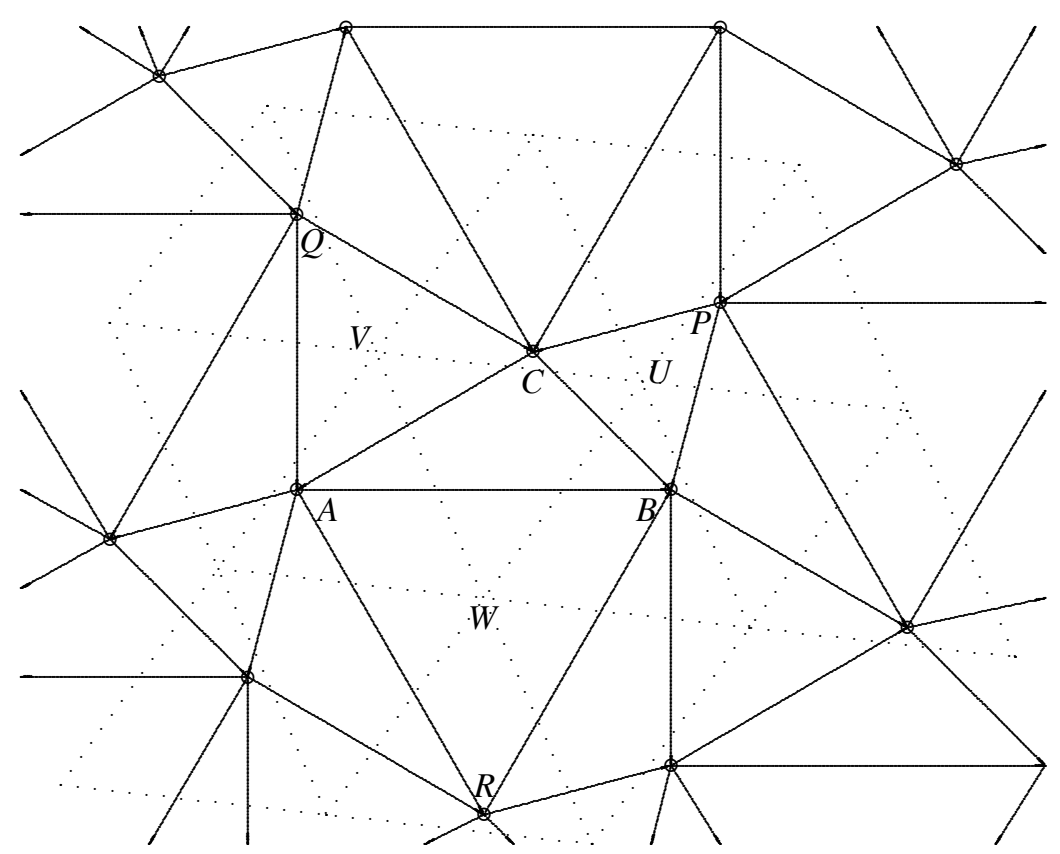

Figure 2

$\psi\left(a^{2} b\right)=\mathbf{T}_{(0,0) \rightarrow(1,-2)}$ and $\psi\left(b a^{2}\right)=\mathbf{T}_{(0,0) \rightarrow(1,1)}$. More generally, $\psi\left[\left(a^{2} b\right)^{t}\left(b a^{2}\right)^{r}\right]=$ $\mathbf{T}_{(0,0) \rightarrow(r+t, r-2 t)}=\mathbf{T}_{(0,0) \rightarrow(1,-2)}^{t} \mathbf{T}_{(0,0) \rightarrow(1,1)}^{r}$. Thus $\psi$ maps $H$ bijectively onto the lattice of translations generated by the vectors $(1,-2)$ and $(1,1)$ (see Figure 3$)$. The note following Theorem 1 together with the simple fact that the rotation $\mathbf{R}_{(0,0)}$ cannot be one of the translations imply that $\psi$ is a bijection.

For completeness, we remark that the elements of $G$ other than those from $H$ belong to one of the following two cosets. The elements with $w(g) \equiv 1 \bmod 3$ correspond to anticlockwise rotations by $\frac{2 \pi}{3}$ :

$$
\psi\left[\left(a^{2} b\right)^{r}\left(b a^{2}\right)^{r+s} a\right]=\psi\left[a\left(a^{2} b\right)^{s}\left(b a^{2}\right)^{-r}\right]=\mathbf{R}(r, s),
$$

while those with $w(g) \equiv 2 \bmod 3$ are mapped to clockwise rotations:

$$
\psi\left[\left(a^{2} b\right)^{-s}\left(b a^{2}\right)^{r} a^{2}\right]=\psi\left[a^{2}\left(a^{2} b\right)^{-r}\left(b a^{2}\right)^{-r-s}\right]=\mathbf{R}^{2}(r, s) .
$$

Observe that $\psi\left[\left(a^{2} b\right)^{t}\left(b a^{2}\right)^{r+t}\right]=\mathbf{T}_{(0,0) \rightarrow(r, r+3 t)}$. All these statements are readily obtained by routine calculations facilitated by the fact that $H$ is abelian, making use of the earlier mentioned identities $\left(a^{2} b\right)^{n} a=a\left(a^{2} b\right)^{-n}\left(b a^{2}\right)^{-n},\left(b a^{2}\right)^{n} a=a\left(a^{2} b\right)^{n}$ and similar ones. Of course, the abelian normal subgroup $H$ consists of all elements whose weight is a multiple of 3 , and they correspond to all translations of $\mathbf{S}_{N}$.

Remark. Theorem 1 follows readily: $H$ corresponds to the (normal) subgroup of all translations of $\mathbf{S}_{N}$ and $K=\left\langle\psi^{-1}\left(\mathbf{R}_{(0,0)}\right)\right\rangle$. 


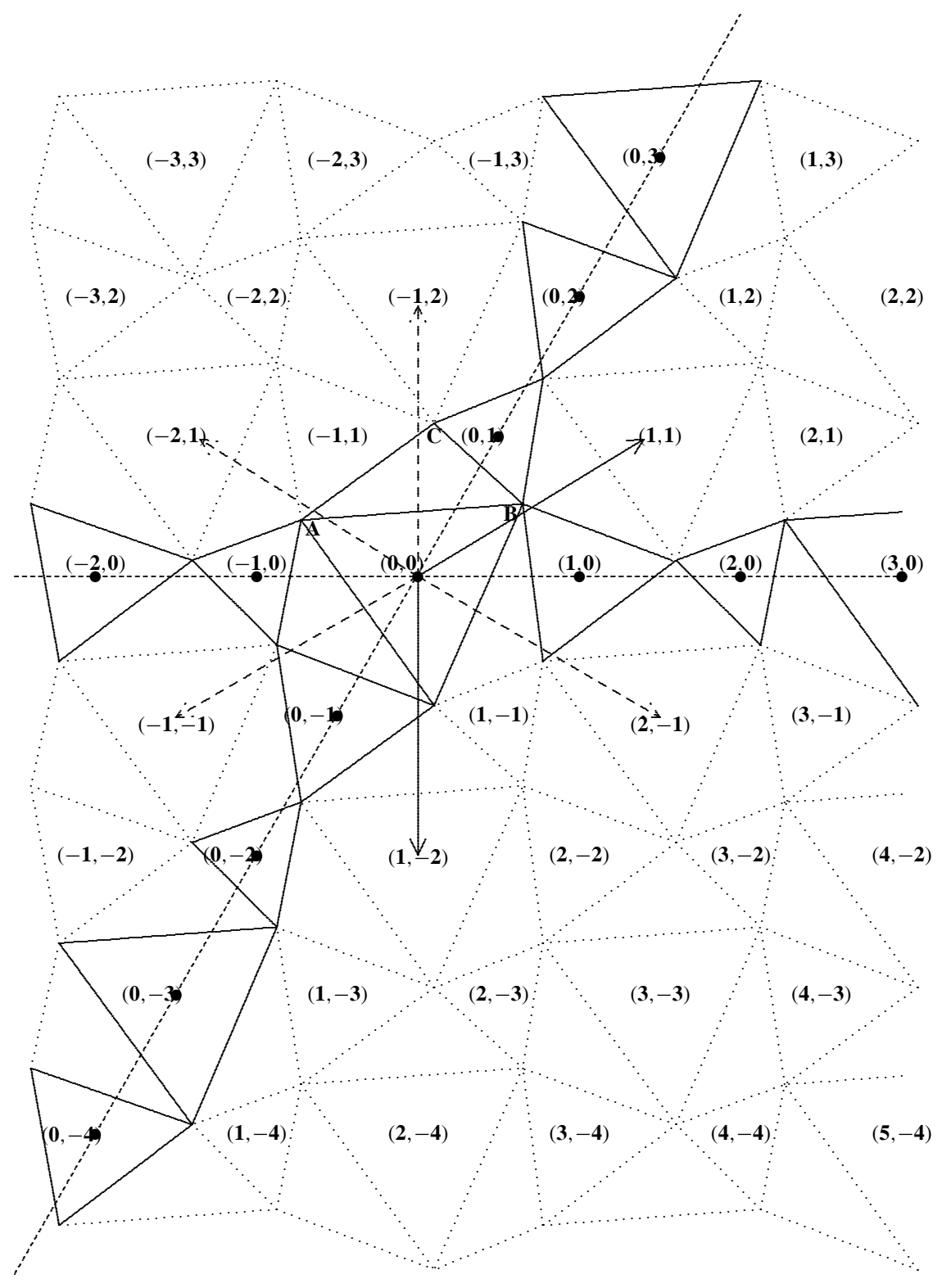

Figure 3

Final remark. The group $G$ is the symmetry group of a scalene Napoleon tessellation: The sides of the triangle $\triangle=A B C$ have different lengths. Indeed, in the case that the triangle is an isosceles triangle (i.e. when $\overline{A C}=\overline{B C} \neq \overline{A B}$ ), the respective symmetry group $\bar{G}=\left\langle b, r \mid b^{3}=r^{2}=\left(r b r b^{2}\right)^{3}=1\right\rangle$ is a semidirect product of the (normal) 
subgroup $G=\left\langle b, r b r b^{2}\right\rangle$ and the cyclic group $\langle r\rangle$ of order 2. Here, using earlier notation, $r b r b^{2}=a$. Furthermore, $r$ corresponds to the reflection with respect to the axis defined by the points $W$ and $C$. Thus there is a tower of normal subgroups: $H=\left\langle(b r b)^{2},\left(b^{2} r\right)^{2}\right\rangle \triangleleft$ $G=\langle b, r b r\rangle \triangleleft \bar{G}$. Observe that $H$ is contained in three subgroups of index 2 in $\bar{G}$. One of these subgroups, viz. $A=H \times\langle r\rangle$ is a maximal abelian subgroup of $\bar{G}$; the other two $\left\langle H, b r b^{2}\right\rangle$ and $\left\langle H, r b r b^{2} r\right\rangle$ are non-commutative. In other words, $\bar{G} \simeq H \rtimes S_{3}$, where $S_{3}=\langle a, r\rangle$.

The group $\bar{G}$ is standardly denoted by $\mathbf{p 3 1 m}$. In case when the "defining" triangle is equilateral, the Napoleon tessallation is just the regular triangular tessellation, and its symmetry group is $\mathbf{p 6 m}$ (see [2]; the reader should beware that the notation in Table 3 differs from the standard one in that the group $\mathbf{p 3 1} \mathbf{m}$ is denoted by $\mathbf{p 3 m} \mathbf{1}$ and vice versa).

Acknowledgement. The author wishes to acknowledge referee's suggestions, as well as fruitful discussions with Dr. Tomáš Pospíchal, that both lead to several improvements in the presentation of the paper.

\title{
References
}

[1] H.S.M. Coxeter, Introduction to Geometry, John Wiley \& Sons, New York - London, 1961.

[2] H.S.M. Coxeter and W.O. Moser, Generators and Relations for Discrete Groups, Ergebnisse der Math. Band 14, Springer-Verlag, New York 1965.

[3] E.S. Fedorov, The Symmetry of Regular Systems of Figures, Proc. St. Petersburg Mineral. Soc. 28 (1891), $1-146$

[Eng. transl. in Symmetry of Crystals, Amer. Crystall. Assoc. Monograph 7 (1971), 50-131].

[4] J.F. Rigby, Napoleon, Escher, and Tessellations, Math. Magazine, 64 (1991), 242-246.

\author{
Vlastimil Dlab \\ Katedra didaktiky matematiky \\ Matematicko-fyzikální fakulta Karlovy university \\ Praha, Czech Republic \\ e-mail: dlab@karlin.mff.cuni.cz \\ and \\ School of Mathematics and Statistics \\ Carleton University \\ Ottawa, Canada \\ e-mail: vdlab@math.carleton.ca
}

